Ion energy and angular distributions onto polymer surfaces delivered by dielectric barrier discharge filaments in air: II. Particles

This article has been downloaded from IOPscience. Please scroll down to see the full text article.

2011 Plasma Sources Sci. Technol. 20035018

(http://iopscience.iop.org/0963-0252/20/3/035018)

View the table of contents for this issue, or go to the journal homepage for more

Download details:

IP Address: 141.211.173.82

The article was downloaded on 06/04/2012 at 16:27

Please note that terms and conditions apply. 


\title{
Ion energy and angular distributions onto polymer surfaces delivered by dielectric barrier discharge filaments in air: II. Particles
}

\author{
Natalia Yu Babaeva and Mark J Kushner ${ }^{1}$ \\ University of Michigan, Department of Electrical Engineering and Computer Science 1301 Beal Ave, \\ Ann Arbor, MI 48109, USA \\ E-mail: nbabaeva@umich.edu and mjkush@umich.edu
}

Received 3 January 2011, in final form 22 March 2011

Published 28 April 2011

Online at stacks.iop.org/PSST/20/035018

\begin{abstract}
Atmospheric pressure streamers intersecting particles are of interest in the context of plasma aided combustion, where the particle may be a fuel aerosol droplet, or in sterilization of air, where the particle may be a bacterium. The ion energy and angular distributions (IEADs) incident on the particles, small curved dielectric surfaces, then in part determine the propensity for activating chemical reactions or, in the case of bacteria, the plasma's sterilization capability. In this paper, we discuss results from a computational investigation of IEADs on small particles ( $45 \mu \mathrm{m}$ radius) produced by atmospheric pressure discharge. Streamers intersecting a particle momentarily generate a large sheath potential as the streamer passes by as the particle charges towards the plasma floating potential. During that time, ions of energies up to $3-10 \mathrm{eV}$ can strike the particle. The permittivity of the particle and the streamer polarity in part determine the character of the IEAD.
\end{abstract}

(Some figures in this article are in colour only in the electronic version)

\section{Introduction}

Atmospheric pressure plasmas (APPs) are used to functionalize polymer surfaces in a wide variety of applications. These applications include commodity products such as polypropylene packaging of biocompatible implants having complex shapes such as tissue scaffolds and drug delivering micro-beads [1-7]. In these surface treatments, the plasma affects only the top few nanometers, modifying the surface characteristics independently of the volume properties of a material. The capability of modifying the surface while not affecting the bulk or structural properties makes APPs promising for processing of biomedical surfaces. These surfaces are often non-planar and rough, as in tissue scaffolding [5-7]. One extreme of non-planarity of such surfaces is biodegradable porous beads as used for drug delivery and gene therapy that are typically tens of $\mu \mathrm{m}$ in diameter. The

\footnotetext{
1 Author to whom any correspondence should be addressed.
}

uniformity of functionalization or deposition on small particles and powders can be improved by suspending them in the gas phase where they are processed by the APP [7-10]. These plasmas are often dielectric barrier discharges (DBDs) which operate in a filamentary mode $[11,12]$.

One of the broad applications of APPs is sterilization and disinfection [13]. The causes of bacteria killing by plasmas are likely many-fold, including UV light, radicals and energetic ion bombardment. Of particular interest is the plasma sterilization of air by killing floating bacteria. Topologically, bacteria floating in air being intersected by APPs is the same problem as functionalization of small polymer beads by plasmas, although there may be different spatial scales. As such, knowing the distribution of ion energies to floating particles is of importance in evaluating the disinfection capability of APPs.

When a streamer in, for example, a DBD strikes a flat dielectric surface whose dimensions are much larger than that of the streamer, the majority of the applied voltage is 
transferred to the sheath at the dielectric surface. As a result, the electric field in the sheath can be many hundreds of $\mathrm{kV} \mathrm{cm}^{-1}$. With mean free paths of the order of $0.5-1 \mu \mathrm{m}$ for ions at atmospheric pressure, one can expect ion energies of up to several tens of $\mathrm{eV}$ incident onto the surface. The ion energy and angular distributions (IEADs) produced by plasma filaments in DBDs striking flat polymer surfaces is the topic of the companion paper, part I [14]. The situation differs when the filament intersects a small, electrically floating dielectric, such as a dust particle, drug delivery bead or bacterium. Depending on the size of the particle relative to the size of the streamer, the plasma may envelope, propagate around or be stopped by the particle [15]. The latter case, that of a large particle or a particle having a large capacitance, mimics that of a streamer intersecting a flat surface and ion energies incident on such a particle are expected to be comparable to those striking a flat surface.

For a particle or bacterium enveloped by the plasma, the electric potential of the particle will ultimately be in equilibrium with the floating potential of the plasma. However, transiently the particle will experience higher potentials. With these transient sheaths, ions with greater than thermal energies can be accelerated onto the particles. In this paper, we report on results from a computational investigation of IEADs incident onto electrically floating dielectric particles. Ion energies incident on floating particles can exceed a few $\mathrm{eV}$ due to the transient sheaths formed around small particles before they equilibrate toward the floating potential. Since the streamer approaches the particle from one side, the IEADs incident on the particle may be asymmetric as a function of position. The description of the geometry and conditions used in this investigation are in section 2. Results for IEADs on particles are discussed in section 3. Our concluding remarks are in section 4

\section{Model, geometry and conditions}

The physical processes, algorithms and computational model used in this investigation are the same as discussed in the companion paper part I [14]. The model, nonPDPSIM, is a two-dimensional, multi-fluid hydrodynamics simulation in which transport equations for all charged and neutral species, Poisson's equation and electron temperature are integrated as a function of time. The electron transport coefficients and rate coefficients as a function of average electron energy are obtained by solving Boltzmann's equation for the electron energy distribution. Poisson's equation is solved throughout the entire computational domain including dielectric materials. Conservation equations for surface and volume charge are solved on and inside all non-metallic materials. Radiation transport and photoionization are addressed using Green's function propagator method. An unstructured numerical mesh using triangular elements with refinement regions was used to resolve the reactor and the particles. The meshes consisted of approximately 7000 nodes, of which more than 5500 are in the plasma region. Ion energies incident onto the particle are computed using a Monte Carlo simulation [16].
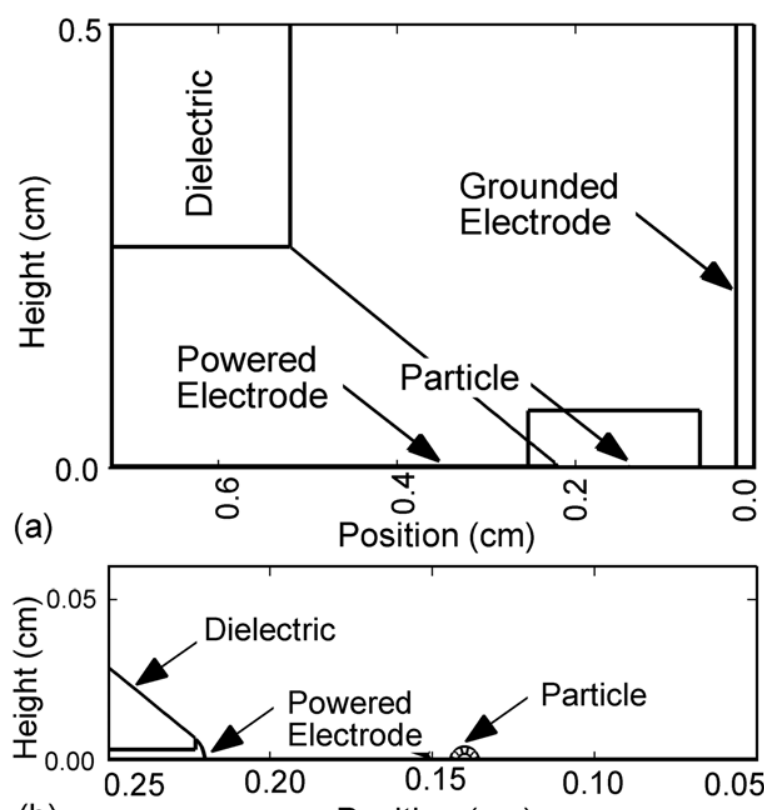

(b) Position (cm)

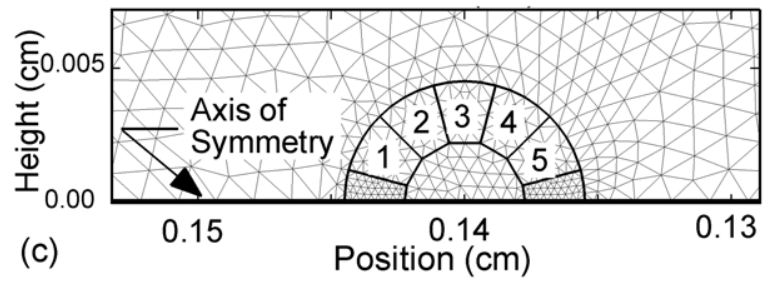

Figure 1. Geometry used for streamer-particle interaction. (a) Total computational domain. (b) Magnification of the point to plane region with a particle. $(c)$ Magnification of the particle covered with the unstructured mesh. Numbers 1 to 5 denote sites on the particle surface where ions are collected.

The geometry used for investigating streamer-particle interaction is shown in figure 1. Positive corona discharges are sustained between a rod encased in a dielectric $\left(\varepsilon / \varepsilon_{0}=3\right)$ biased to $15 \mathrm{kV}$ and separated from a flat grounded electrode (not covered by dielectric) by $2 \mathrm{~mm}$. A particle $45 \mu \mathrm{m}$ in radius is suspended approximately in the middle of the gap. The surface sites where IEADs are recorded are labeled 1 to 5 in figure $1(c)$. To initiate streamers, a small spot of seedcharges (electrons and $\mathrm{N}_{2}^{+}$with peak density of $10^{8} \mathrm{~cm}^{-3}$ and diameter $250 \mu \mathrm{m}$ ) was placed $10 \mu \mathrm{m}$ from the tip of the anode. There was otherwise no initial plasma density elsewhere in the computational domain.

\section{IEADs incident onto floating particles}

The dynamics of streamers intersecting with dielectric particles are discussed in detail in [15]. Briefly, a streamer intersecting a particle of similar or larger radius can be absorbed by the particle while the capacitance of the particle is charged by electron and ion fluxes from the streamer to the particle. Once the particle fully charges, the streamer can be reinitiated from the opposite side of the particle seeded by the same photoionization that sustains the streamer or by plasma that flows around the particle. Positive streamers are more sensitive to this effect than negative streamers. The larger the dielectric 
Positive Streamer, $\varepsilon / \varepsilon_{0}=2$

$\mathrm{O}_{2}^{+}$density $\left(\mathrm{cm}^{-3}\right)(3 \mathrm{dec}) \quad$ Close-up

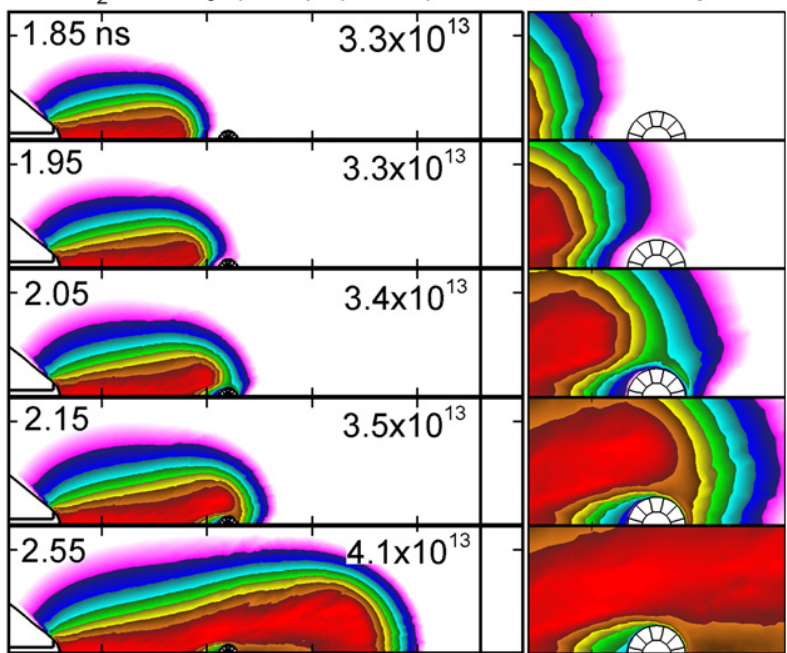

$\rho\left(\mathrm{cm}^{-3}\right)(2 \mathrm{dec})$

Close-up

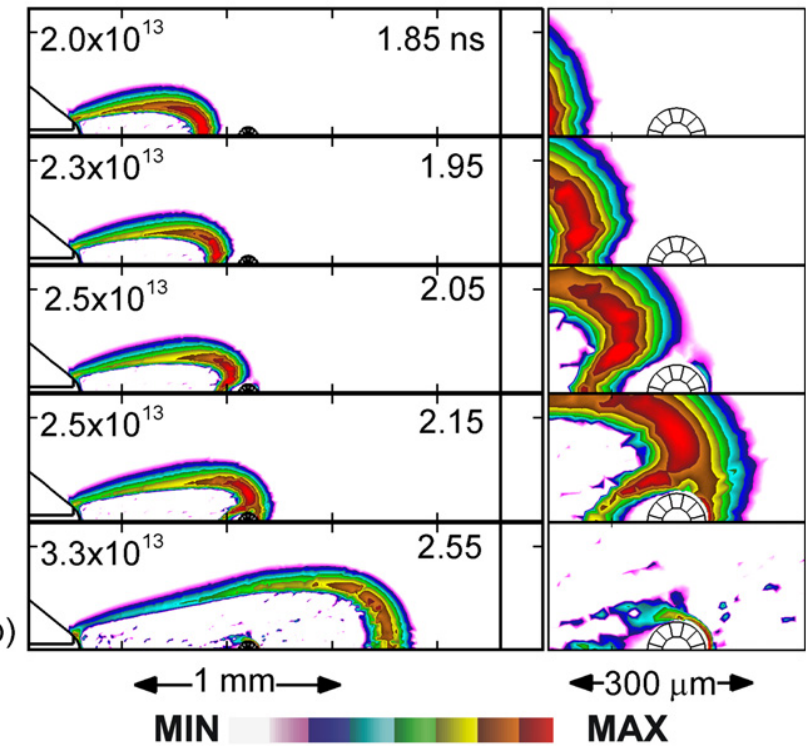

Figure 2. Positive streamer $(15 \mathrm{kV})$ enveloping a small dielectric $\left(\varepsilon / \varepsilon_{0}=2\right)$ particle $45 \mu \mathrm{m}$ in radius as a function of time. $(a) \mathrm{O}_{2}^{+}$ion density and $(b)$ positive space charge. The contours are log-scales with number of decades and maximum values noted in the frame. Some amount of charge is removed from the streamer by the particle. Wakes form both upstream and downstream.

constant of the particle, the more charged flux from the streamer is required to charge the particle, and so the greater the perturbation in the dynamics of the streamer. If the streamer radius is larger than that of a particle having a low dielectric constant, the streamer envelopes the particle, charging it toward the floating potential. As the streamer passes the particle, the particle is largely left in electrical equilibrium with the conductive channel trailing the streamer head, charged to the floating potential. Polarization of the particle produces a maximum in electric field at the poles of the particle along the axis of the discharge. There are minima in electric fields at the equator.

The $\mathrm{O}_{2}^{+}$density and positive charge for a positive streamer $(15 \mathrm{kV})$ intersecting a $45 \mu \mathrm{m}$ radius particle with $\varepsilon / \varepsilon_{0}=2$ are (a)
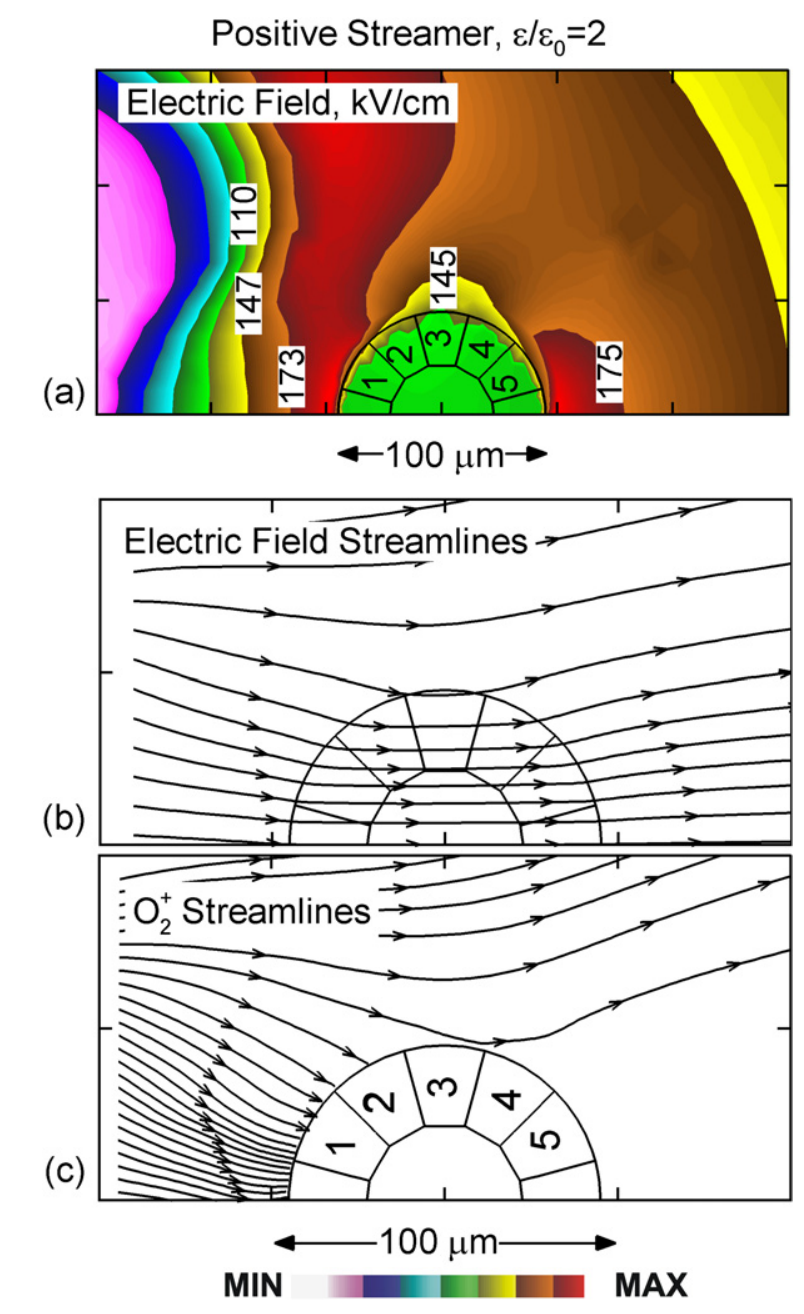

Figure 3. Plasma properties at $t=2.05 \mathrm{~ns}$ when the streamer tip approaches and envelopes the particle $\left(\varepsilon / \varepsilon_{0}=2\right)$ for a positive streamer $(15 \mathrm{kV})$. (a) Electric field, (b) electric field streamlines and (c) streamlines of the $\mathrm{O}_{2}^{+}$ion flux. The low dielectric constant particle is moderately polarized with enhancement of the electric field at the poles where electric field streamlines converge. The top portion of particle receives higher ion fluxes which impinge almost normally onto site 1 .

shown in figure 2. The electric field magnitude, streamlines and $\mathrm{O}_{2}^{+}$flux streamlines are shown in figure 3 . Note that the streamlines in figure 3 show the direction of the vector fields but not their magnitude. As the radius of the streamer is large compared with the radius of the particle, the streamer envelopes the particle. With electron densities of $4 \times 10^{13} \mathrm{~cm}^{-3}$, the Debye length is only $2 \mu \mathrm{m}$, and so a sheath forms around the periphery of the particle. The direction of positive ion drift is from left-to-right in figure 2 and the drift of electrons is right to left. There are two wakes that are formed as plasma flows by the particle. The major wake is to the right in the direction of propagation of the streamer. Photoionization is the mechanism whereby a positive steamer propagates into non-ionized gas. The shadowing of photoionization by the particle produces a wake of diminished ion density downstream of the particle. The wake is also formed by the shadowing of streaming ions originating upstream of the particle. The smaller upstream wake results from the blocking of the more rapid electron fluxes from the downstream side of the particle. 

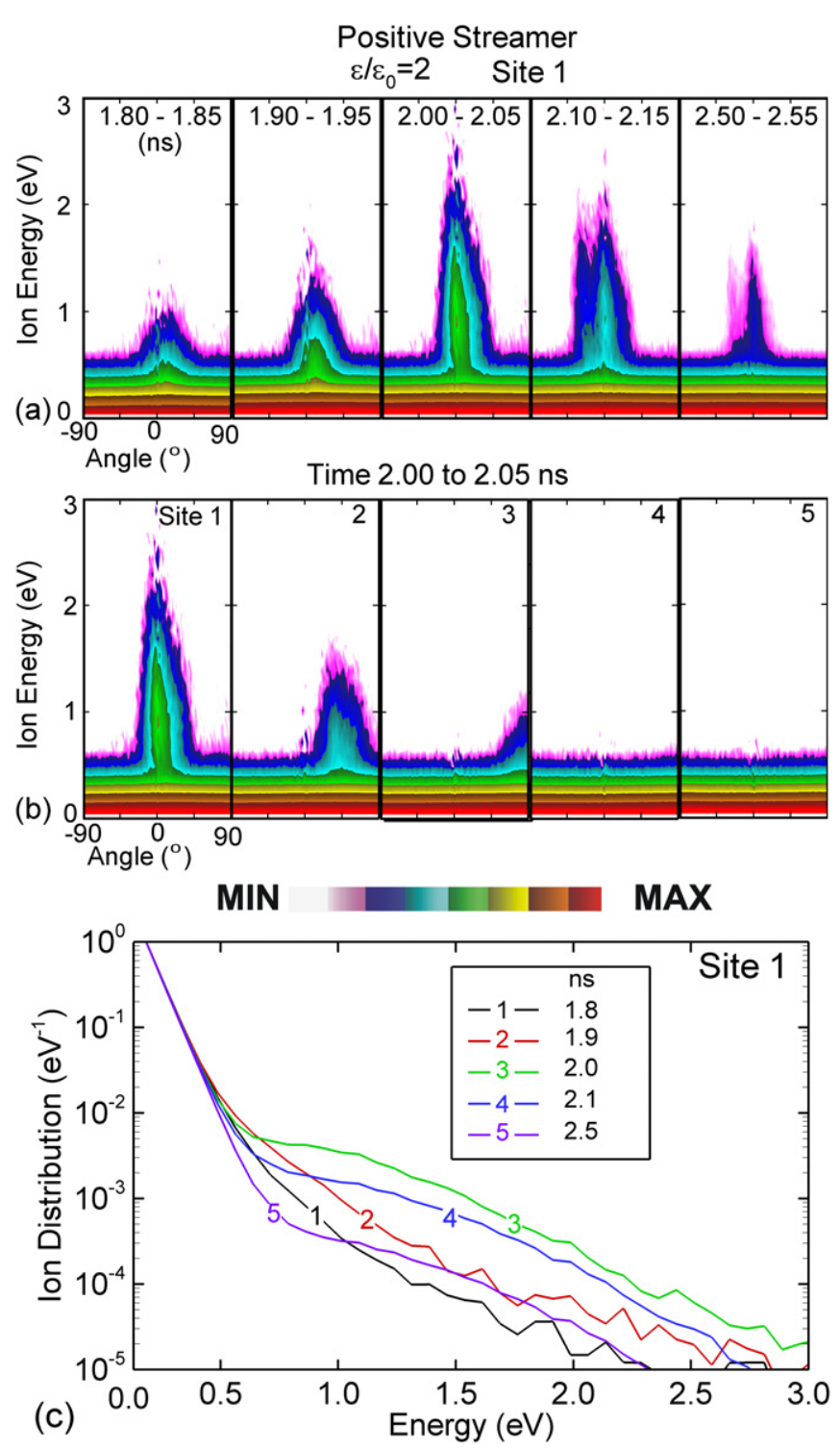

Figure 4. IEADs onto a particle $\left(\varepsilon / \varepsilon_{0}=2\right)$ for a positive streamer $(15 \mathrm{kV})$. (a) IEADs onto site 1 for times corresponding to streamer positions in figure 2. (b) IEADs onto sites $1-5$ for $t=2.0 \mathrm{~ns}$. (c) Ion energy distributions integrated over angle for site 1 for different times. Two groups of ions are recorded. The first group is nearly isotropic thermalized ions with energies below $1 \mathrm{eV}$. The second group is fast ions with energies up to $3 \mathrm{eV}$, accelerated by sheath electric fields nearly normal to the surface. Contours are plotted over 4 decades on a log-scale.

The low dielectric constant particle is only moderately polarized in the applied and space charge electric fields with an enhancement of the electric field at the poles of the particle up to $175 \mathrm{kV} \mathrm{cm}^{-1}$ as shown in figure 3 . With this polarization and convergence of the electric field at the poles, the $\mathrm{O}_{2}^{+}$streamlines are also moderately focused into the top of the particle (sites 1 and 2). There is little net flux of $\mathrm{O}_{2}^{+}$onto the bottom of the particle (sites 4 and 5). As the avalanche front of the streamer passes the particle, there is a finite time (typically many nanoseconds) during which the particle charges to the floating potential [17]. As such, it is unlikely that a particle attains the full floating potential during the streamer propagation times of a few ns.
Positive Streamer, $\varepsilon / \varepsilon_{0}=80$
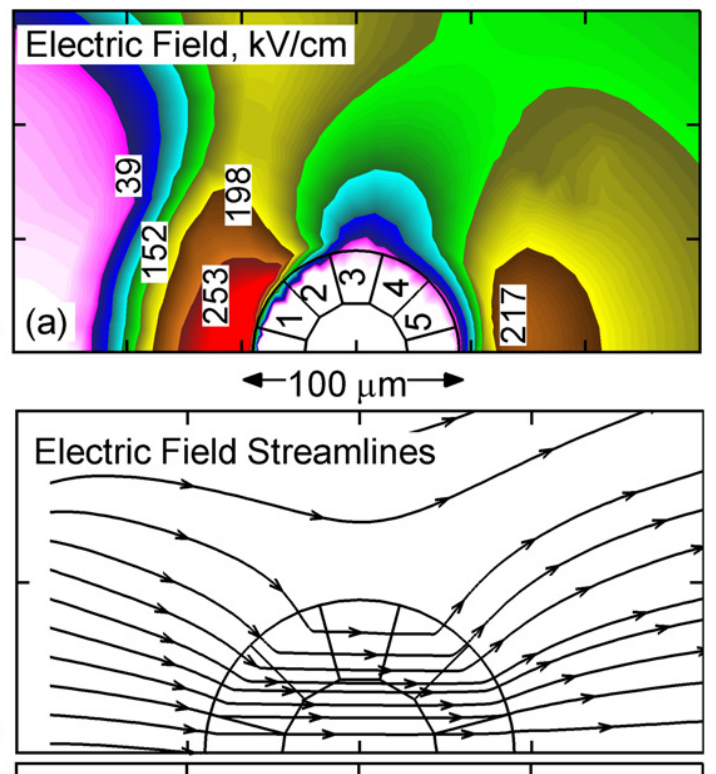

(b)

(c)

\section{$\mathrm{O}_{2}^{+}$Streamlines}

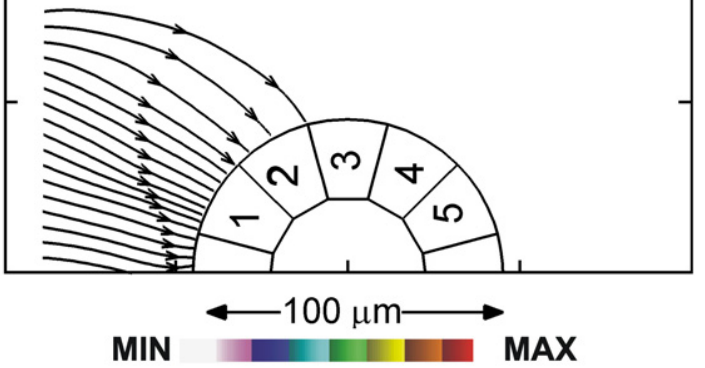

Figure 5. Plasma properties at $t=2.05 \mathrm{~ns}$ when the streamer tip approaches and envelopes a high dielectric constant particle $\left(\varepsilon / \varepsilon_{0}=80\right)$ for a positive streamer $(15 \mathrm{kV})$. (a) Electric field, (b) electric field streamlines and $(c)$ streamlines of $\mathrm{O}_{2}^{+}$ion flux. The particle with high dielectric constant is highly polarized with enhancement of the electric field at the poles. The high refraction of the electric field lines at the particle boundary produces a more normal incidence of ions onto the particle surface.

The IEADs for $\mathrm{O}_{2}^{+}$incident on the particle at site 1 are shown in figure 4(a). The IEADs are averaged over a period of $\Delta t=0.05 \mathrm{~ns}$ corresponding to the streamer positions shown in figure 2. The IEADs consist of two groups of ions. The first group has thermalized ions with energies below $1 \mathrm{eV}$ arriving with essentially isotropic trajectories. The temperature of the thermal ions is about $0.1 \mathrm{eV}$. The second group consists of fast ions with energies up to $2-3 \mathrm{eV}$. These ions arrive with nearly normal incidence, accelerated by electric field vectors that are also near normal to the surface. At $t=2.0 \mathrm{~ns}$, the IEAD on site 1 has the highest ion energy, corresponding to the peak in the electric field, and in this case is angularly symmetric with respect to the local normal. The maximum electric field at the surface of the particle at this time is $175 \mathrm{kV} \mathrm{cm}^{-1}$ (or $E / N$ (electric field/gas density) $=700 \mathrm{Td}, 1 \mathrm{Td}=10^{-17} \mathrm{~V} \mathrm{~cm}^{-2}$ ). For a mean free path of $0.5 \mu \mathrm{m}$ in an average electric field of $150 \mathrm{kV} \mathrm{cm}^{-1}$, expected ion energies are around $3-4 \mathrm{eV}$, commensurate with that predicted here.

The IEADs incident on sites $1-5$ at $t=2.0 \mathrm{~ns}$ are shown in figure $4(b)$. While the upper surface (site 1 ) receives an impulse 

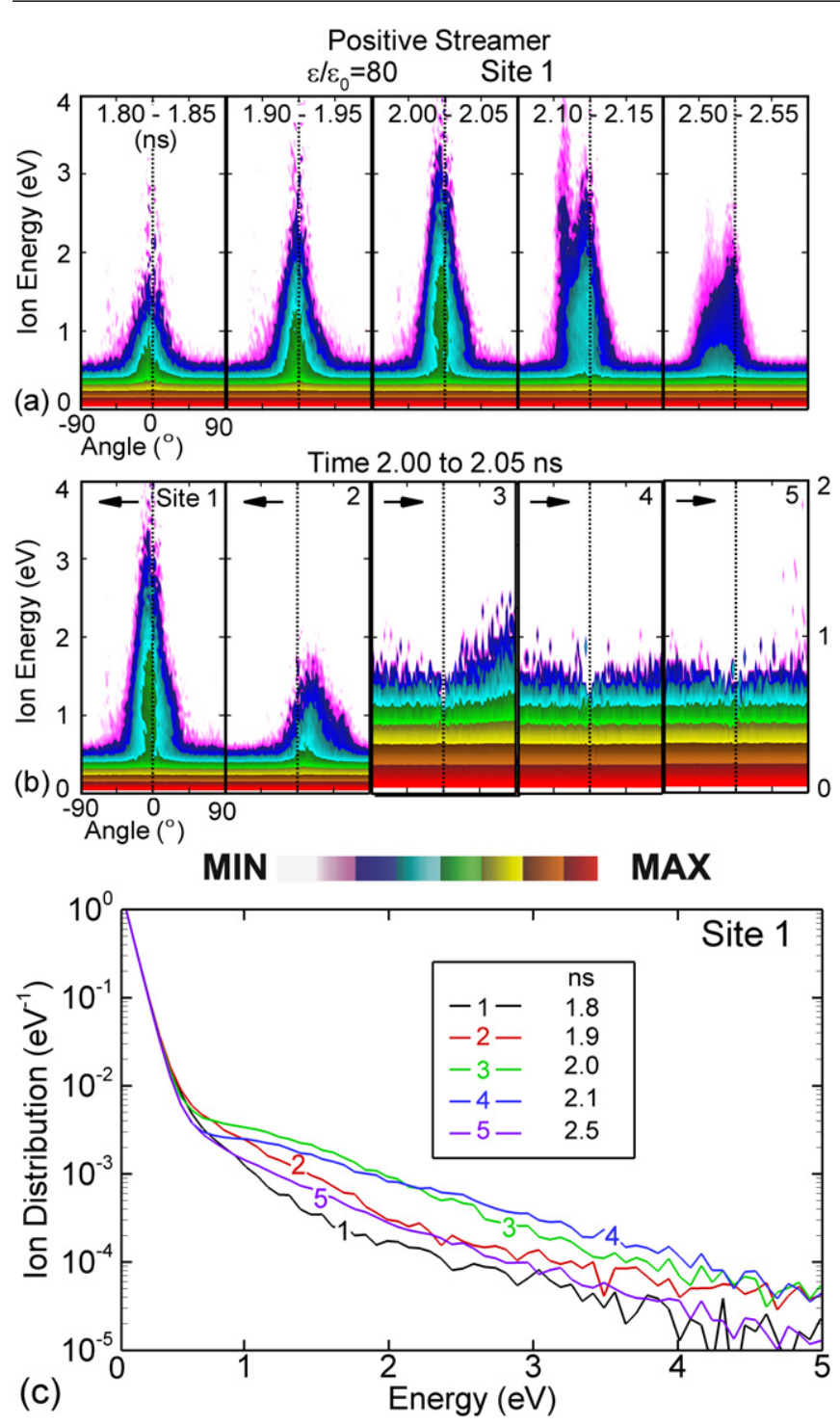

Figure 6. IEADs on a high dielectric constant particle $\left(\varepsilon / \varepsilon_{0}=80\right)$ for a positive streamer $(15 \mathrm{kV})$. (a) IEADs on site 1 as a function of time. (b) IEADs on sites $1-5$ for $t=2.05 \mathrm{~ns}$. Arrows indicate which energy scale is used. $(c)$ Ion energy distributions integrated over angle for site 1 for different times. At site 1 ion energies are symmetric and higher energy compared with the low permittivity particle. For site 2, the IEAD is asymmetric, shifted to the right, with less energetic ions compared with site 1 . Site 3 receives almost no energetic ions and the majority of the ions are thermalized. Contours are plotted over 4 decades on a log-scale.

of energetic ions, ions arriving at sites 2 and 3 are progressively less energetic. The non-thermal ions arrive at these sites with progressively less vertical and more grazing angles, which reflect the direction of the electric field intersecting the particle (see figure 3). Ions arriving on the bottom surfaces (sites 4 and 5) are essentially thermalized. The direction of the electric field points away from the surface at the time of peak electric field, and so only thermal ions produced within a few mean free paths of the surface will strike the surface. Ion energy distributions for site 1 integrated over angle are shown in figure $4(c)$ as a function of time. Only $1 \%$ of ions have energies that exceed $0.5 \mathrm{eV}$.

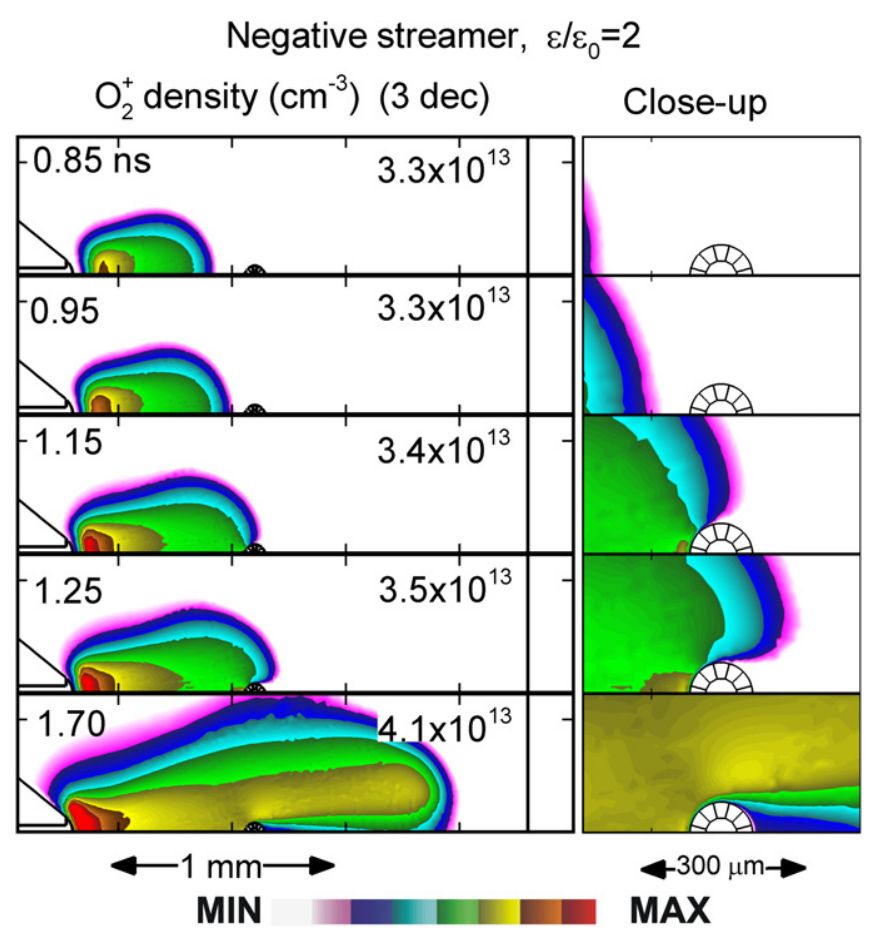

Figure 7. $\mathrm{O}_{2}^{+}$ion density as a negative streamer $(-15 \mathrm{kV})$ envelopes a small dielectric $\left(\varepsilon / \varepsilon_{0}=2\right)$ particle of $45 \mu \mathrm{m}$ radius as a function of time. A wake forms downstream of the particle due to shadowing of electrons drifting in the same direction as the direction of the streamer.

The shape, size and permittivity of the particle in part determine the character of the IEAD. In particular, the permittivity of the particle and its size relative to the streamer determine the character of the streamer-particle interaction, as discussed in [15]. For example, the electric field and streamlines for the electric field and flux of $\mathrm{O}_{2}^{+}$ions are shown in figure 5 for a particle having $\varepsilon / \varepsilon_{0}=80$, akin to a water droplet. The timing is at $t=2.05 \mathrm{~ns}$ as the tip of the streamer approaches the particle. A particle with a larger dielectric constant is more highly polarized with more severe enhancement of the electric field at the poles of the particle. The maximum electric field at the pole of the particle is $250 \mathrm{kV} \mathrm{cm}^{-1}$ (1000 Td), compared with $175 \mathrm{kV} \mathrm{cm}^{-1}$ (700 Td) for a particle with $\varepsilon / \varepsilon_{0}=2.2$. Since the capacitance of the particle scales with the dielectric constant, a longer period is required to charge the particle to the plasma potential, which produces normal electric fields at the surface of longer duration and greater magnitude. Electric field and $\mathrm{O}_{2}^{+}$streamlines are more focused at the poles due to the larger polarization, which results in a more normal angle of incidence of the ions.

IEADs incident on site 1 for $\varepsilon / \varepsilon_{0}=80$ particle are shown in figure $6(a)$ as the streamer passes and charges the particle. The angular distributions are nearly symmetric to the normal, a consequence of the refraction of the electric fields to near normal incidence by the particle polarization. The larger sheath potential $(\sim 10 \mathrm{~V}$ compared with $\sim 7 \mathrm{~V}$ for $\varepsilon / \varepsilon_{0}=2.2$ ) for a longer duration produces ion energies up to $4 \mathrm{eV}$ on site 1 , roughly twice that of the particle having $\varepsilon / \varepsilon_{0}=2$. IEADs for sites $1-5$ are shown in figure $6(b)$ at $t=2.0-2.05 \mathrm{~ns}$. At site 2, the IEAD is angularly asymmetric, 
(a)

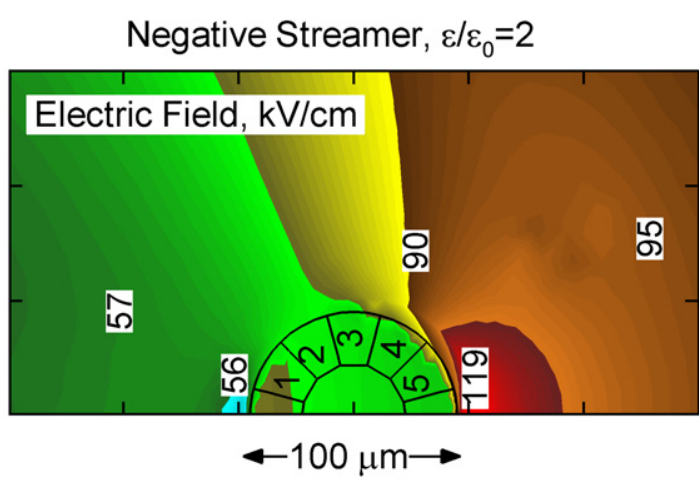

(b)

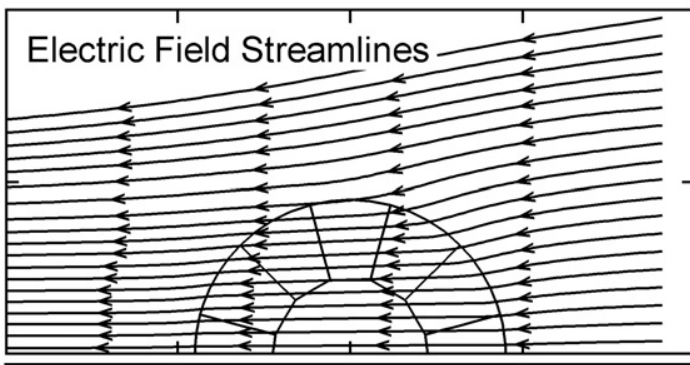

(c)

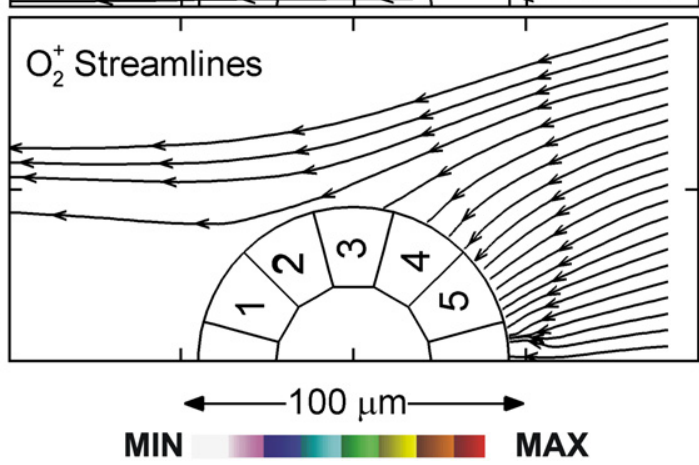

Figure 8. Plasma properties at $t=1.25 \mathrm{~ns}$ when the streamer tip approaches and envelopes the particle $\left(\varepsilon / \varepsilon_{0}=2\right)$ for a negative streamer $(-15 \mathrm{kV})$. (a) Electric field, $(b)$ electric field streamlines and $(c)$ streamlines of $\mathrm{O}_{2}^{+}$ion flux. All characteristics for electric field, electric field streamlines and ion flux streamlines are similar to those shown in figure 3 for the positive streamer. The primary difference is the reversal of the direction of the electric field and drift of ions.

shifted to the right with peak energies of about $1.5 \mathrm{eV}$. Sites $3-5$ receive almost no ions with greater than thermal energies as the majority of the ions are directed to the top surfaces facing the approaching streamer. For a particle with $\varepsilon / \varepsilon_{0}=2.2$, site 3 received ions of up to $1 \mathrm{eV}$, albeit at grazing angles. Ion energy distributions for site 1 integrated over angle are shown in figure 4(c). These distributions qualitatively resemble those for the lower permittivity particle, though with a more extended tail. Only $1 \%$ of ions exceed an energy of $0.8 \mathrm{eV}$.

In DBDs, particles may experience alternate pulses of what appear to be negative and positive streamers. The density of $\mathrm{O}_{2}^{+}$is shown in figure 7 for a negative streamer with voltage $-15 \mathrm{kV}$. Negative streamers are typically wider and more diffuse than positive streamers and electron drift is in the direction of propagation of the streamer (left to right in the figure). The $45 \mu \mathrm{m}$ particle is fully enveloped by the streamer. The electron density in the vicinity of the streamer is $1.1 \times 10^{13} \mathrm{~cm}^{-3}$, producing a sheath thickness of $4 \mu \mathrm{m}$ surrounding the particle. The more rapid drift of

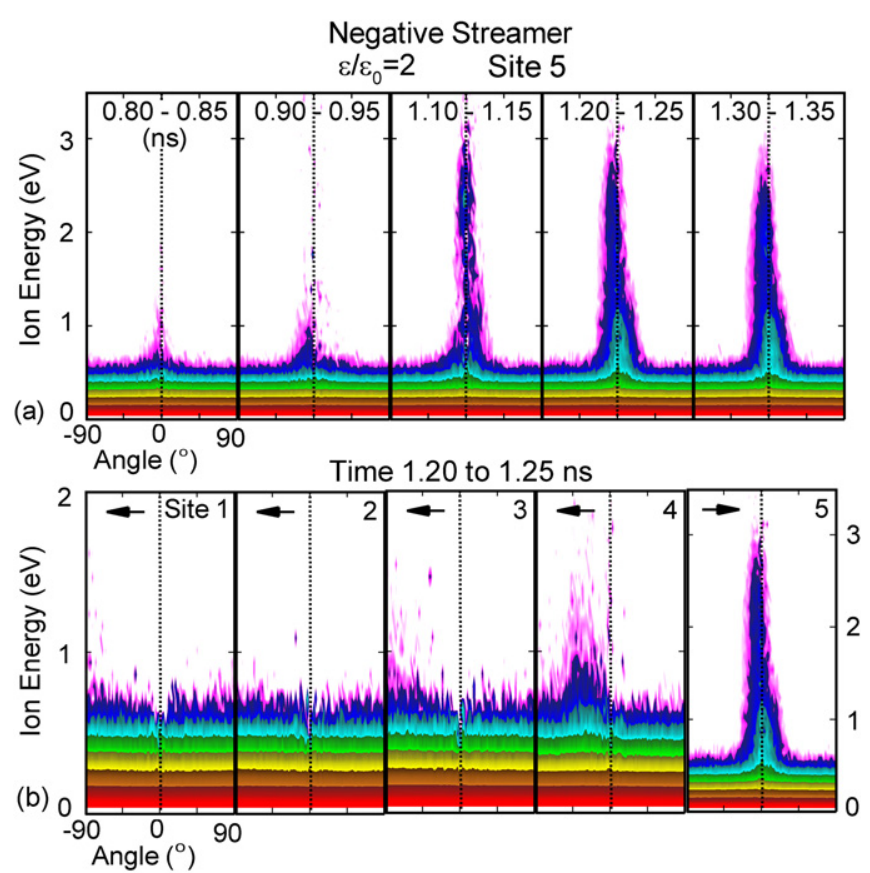

MIN

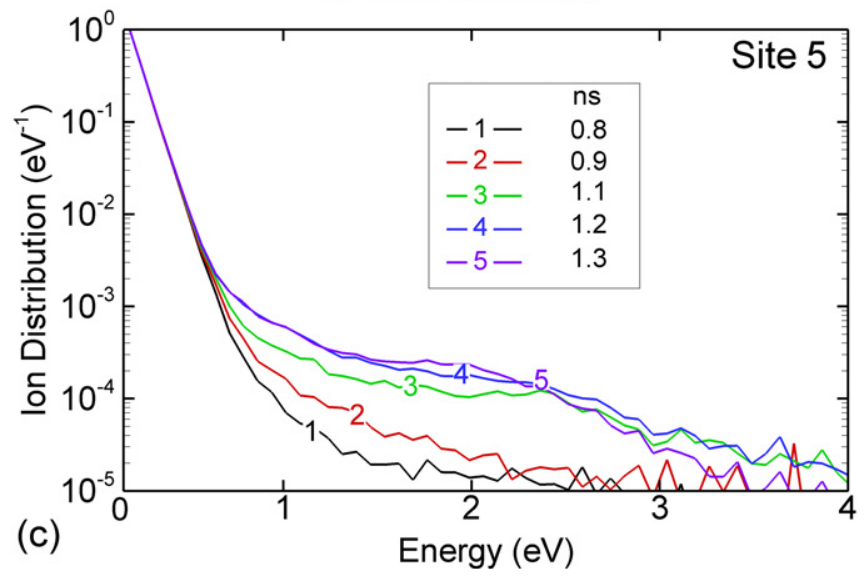

Figure 9. IEADs on a particle $\left(\varepsilon / \varepsilon_{0}=2\right)$ for negative streamer $(-15 \mathrm{kV})$. (a) IEADs on site 5 as a function of time. (b) IEADs on sites $1-5$ for $t=1.2 \mathrm{~ns}$. Arrows indicate which energy scale is used. (c) Ion energy distributions integrated over angle for site 5 for different times. The IEADs mirror those shown in figure 4 for the negative streamer though with perhaps more vertical incidence. Contours are plotted over 4 decades on a log-scale.

electrons compared with ions produces a more extensive wake underneath the particle compared with the positive streamer.

The electric field, and electric field and $\mathrm{O}_{2}^{+}$streamlines are shown in figure 8 for $t=1.25 \mathrm{~ns}$ for the negative streamer. The largest electric field enhancement $\left(120 \mathrm{kV} \mathrm{cm}^{-1}\right.$ or $\left.480 \mathrm{Td}\right)$ occurs on the bottom of the particle where the sheath potential is around 6-7 V. The bottom of the particle is now exposed to fluxes of energetic positive ions. Since the more mobile electrons drift into the particle, the charging of the particle is more rapid. In general, most characteristics for the electric field, and electric field and ion flux streamlines discussed for the positive streamer apply to this case. The primary difference is in the reversed direction of the electric field.

IEADs are shown in figure $9(a)$ for site 5 (bottom of the particle) for times corresponding to the streamer positions in 
(a)

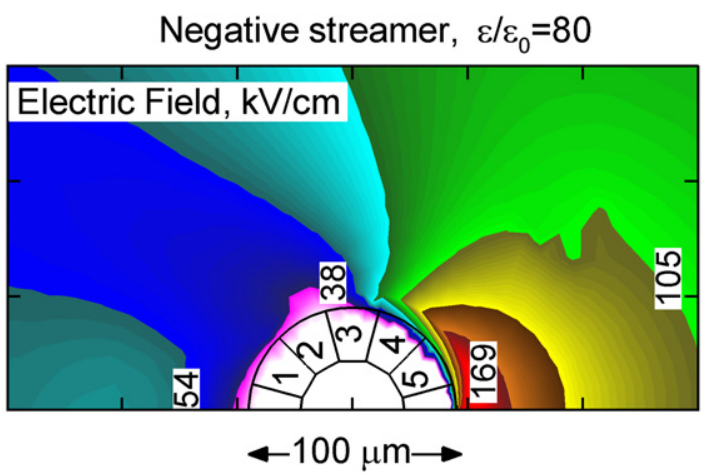

(b)

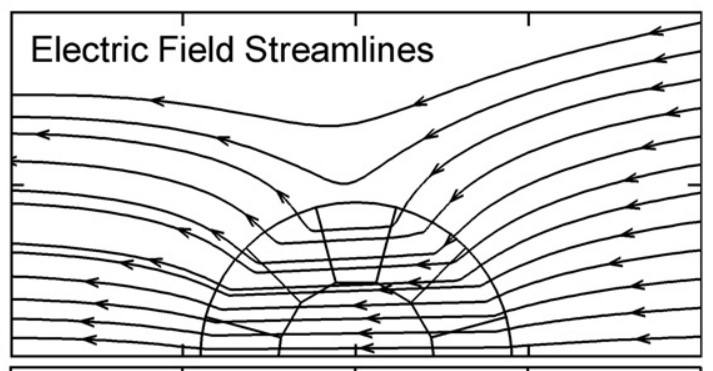

(c)

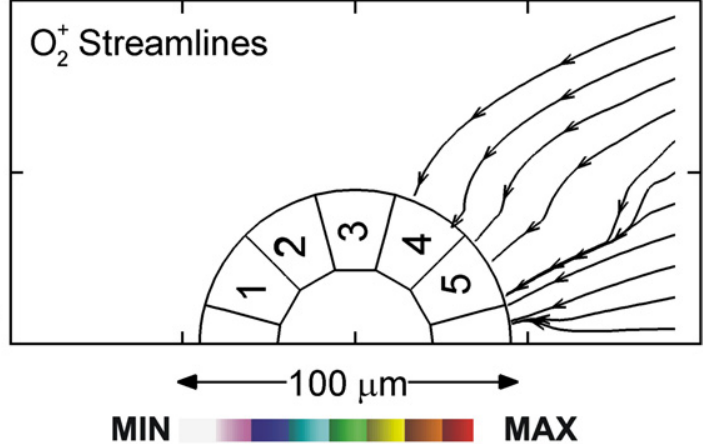

Figure 10. Plasma properties at $t=1.25 \mathrm{~ns}$ when the streamer tip approaches and envelopes a high dielectric constant particle $\left(\varepsilon / \varepsilon_{0}=80\right)$ for a negative streamer $(-15 \mathrm{kV})$. (a) Electric field, $(b)$ electric field streamlines and $(c)$ streamlines of $\mathrm{O}_{2}^{+}$ion flux. All characteristics for electric field, electric field streamlines and ion flux streamlines are similar to those shown in figure 5 for the positive streamer. The primary difference is in the reversal of the direction of the electric field and direction of ion drift.

figure 7. Again, the IEAD consists of two groups of ionsthermal ions with isotropic angular distributions and energetic ions up to $3 \mathrm{eV}$ with narrow angular distributions at near normal incidence. IEADs incident on sites $1-5$ are shown in figure $9(b)$ for $t=1.2 \mathrm{~ns}$. The distributions are nearly mirror images of the IEADs shown in figure 4 for the positive streamer. Site 5 on the bottom surface receives ions with energies up to $3 \mathrm{eV}$ at normal incidence. The ions arrive at progressively grazing angles and lower energies at sites 4 and 3 . The top surfaces receive only thermalized ions. Ion energy distributions for site 5 integrated over angle are shown in figure $9(c)$. As for the positive streamer, only $1 \%$ of ions exceed an energy of $0.5 \mathrm{eV}$. In general, the pulse of energetic ions for negative streamers is shorter and higher in energy by about $1 \mathrm{eV}$ compared with the positive streamer. This results from the more rapid charging of the particle with a thinner sheath.

Images for electric field lines and ion fluxes for a negative streamer enveloping the particle $\varepsilon / \varepsilon_{0}=80$, as shown in
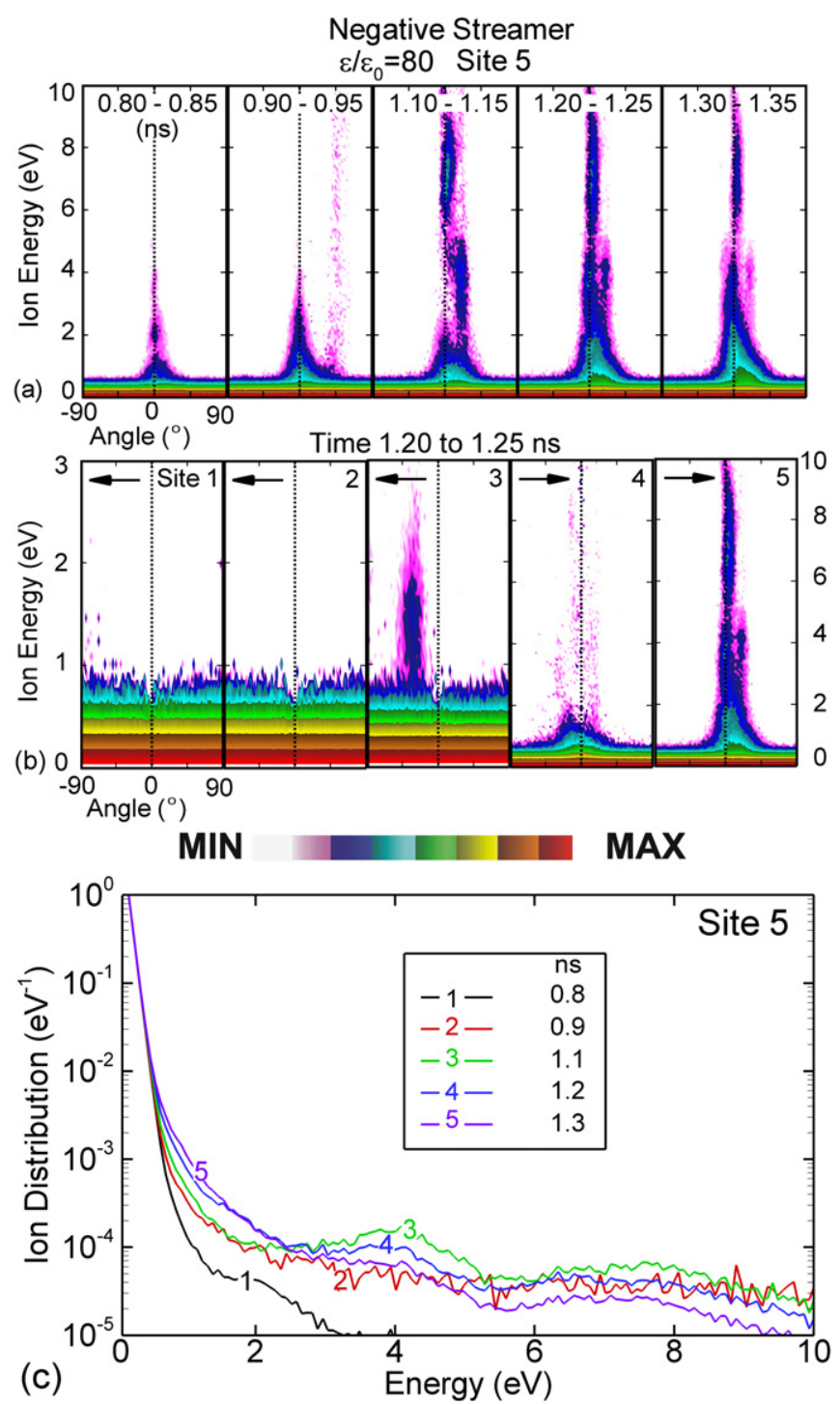

Figure 11. IEADs on a high dielectric constant particle $\left(\varepsilon / \varepsilon_{0}=80\right)$ for a negative streamer $(-15 \mathrm{kV})$. (a) IEADs on site 5 as a function of time. (b) IEADs on sites $1-5$ for $t=1.25 \mathrm{~ns}$. Arrows indicate which energy scale is used. $(c)$ Ion energy distributions integrated over angle for site 5 for different times. Contours are plotted over 4 decades on a log-scale.

figure 10, are similar to those of a positive streamer. Electric field and $\mathrm{O}_{2}^{+}$streamlines are more focused at the poles due to the larger polarization, which results in a more normal angle of incidence of the ions. The main difference with the positive streamer is in the reversed direction of the electric field. Maximum electric fields are $170 \mathrm{kV} \mathrm{cm}^{-1}(680 \mathrm{Td})$ with a sheath potential of $10-12 \mathrm{~V}$. IEADs incident on site 5 for the $\varepsilon / \varepsilon_{0}=80$ particle are shown in figure 11(a). The angular distributions are nearly symmetric to the normal while the larger sheath potential produces ion energies up to $10 \mathrm{eV}$. IEADs for sites $1-5$ are shown in figure $11(b)$ at $t=1.25 \mathrm{~ns}$. At site 4 , the IEAD is angularly asymmetric, slightly shifted to the left with peak energies of about $1.5 \mathrm{eV}$. Sites 3 to 1 receive almost no ions with greater than thermal energies as the majority of the ions are directed to the top surfaces facing the approaching streamer. Ion distributions for site 5 integrated 
over angle are shown in figure $11(c)$. Only $1 \%$ of ions exceed an energy of $0.3 \mathrm{eV}$.

\section{Concluding remarks}

Small particles in the paths of atmospheric pressure plasma streamers transiently charge toward the floating potential. During this transient as the head of the streamer strikes the particle, electric fields $100-200 \mathrm{kV} \mathrm{cm}^{-1}$ are produced near the surface of the particle. With mean free paths of $0.5-1 \mu \mathrm{m}$, ion energies of a few eV can be incident on the particle. Two groups of ions typically strike the particle: thermal low energyhigh angle ions and energetic ions up to 3 to $10 \mathrm{eV}$ depending on the dielectric constant of the particle and polarity of the discharge. These energies occur for only a few ns at most, the time it takes to charge the capacitance of the particle. The energetic ions are distributed asymmetrically on the particle, being focused into the pole of the particle intersecting the ion drift where electric field enhancement is largest. Although less than a percent of ions during this short time is in the energetic group, they may be able to initiate processes that have threshold energies which are not accessible to thermal ions.

\section{Acknowledgment}

This work was supported by the Department of Energy Office of Fusion Energy Science.

\section{References}

[1] Sefton M V, Sawyer A, Gorbet M, Black J P, Cheng E, Gemmell C and Pottinger-Cooper E 2001 Does surface chemistry affect thrombogenicity of surface modified polymers? J. Biomed. Mater. Res. 55447

[2] Koenig U, Nitschke M, Menning A, Sperling C, Simon E, Arnhold C, Werner C and Jacobasch H J 1999 Plasma modification of polytetrafluoroethylene for immobilization of the fibrinolytic protein urokinase Surf. Coat. Technol. 1161011

[3] Upadhyay D J, Cui N-Y, Meenan B J and Brown N M D 2005 The effect of dielectric barrier discharge configuration on the surface modification of aromatic polymers $J$. Phys. D: Appl. Phys. 38922

[4] Borcia G, Chiper A and Rusu I 2006 Using a $\mathrm{He}+\mathrm{N}_{2}$ dielectric barrier discharge for the modification of polymer surface properties Plasma Sources Sci. Technol. 15849

[5] Kim J W and Choi H S 2002 Surface crosslinking of high-density polyethylene beads in a modified plasma reactor J. Appl. Polym. Sci. 832921

[6] Sipehia R, Chawla A S and Chang T M S 1986 Enhanced albumin binding to polypropylene beads via anhydrous ammonia gaseous plasma Biomaterials 7471

[7] Bhoj A N and Kushner M J 2008 Repetitively pulsed atmospheric pressure discharge treatment of rough polymer surfaces: II. Treatment of micro-beads in $\mathrm{He} / \mathrm{NH}_{3} / \mathrm{H}_{2} \mathrm{O}$ and $\mathrm{He} / \mathrm{O}_{2} / \mathrm{H}_{2} \mathrm{O}$ mixtures Plasma Sources Sci. Technol. 17035025

[8] Bretagnol F, Tatoulian M, Arefi-Khonsari F, Lorang G and Amouroux J 2004 Surface modification of polyethylene powder by nitrogen and ammonia low pressure plasma in a fluidized bed reactor React. Funct. Polym. 61221

[9] Sharma R, Trigwell S, Biris A S, Sims R A and Mazumder M K 2003 Effect of ambient relative humidity and surface modification on the charge decay properties of polymer powders in powder coating IEEE Trans. Indust. Appl. 3987

[10] Nessim C, Boulos M and Kogelschatz U 2009 In-flight coating of nanoparticles in atmospheric-pressure DBD torch plasmas Eur. Phys. J. Appl. Phys. 4722819

[11] Kogelschatz U 2003 Dielectric-barrier discharges: their history, discharge physics and industrial applications Plasma Chem. Plasma Process. 231

[12] Kogelschatz U 2010 Collective phenomena in volume and surface barrier discharges J. Phys: Conf. Ser. 257012015

[13] Laroussi M, Mendis D A and Rosenberg M 2003 Plasma interaction with microbes New J. Phys. 541

[14] Babaeva N Yu and Kushner M J 2011 Ion energy and angular distributions onto polymer surfaces delivered by dielectric barrier discharge filaments in air: I. Flat surfaces Plasma Sources Sci. Technol. 20035017

[15] Babaeva N Yu, Bhoj A N and Kushner M J 2006 Streamer dynamics in gases containing dust particles Plasma Sources Sci. Technol. 15591

[16] Babaeva N Yu and Kushner M J 2008 Ion energy and angular distributions into the wafer focus ring gap in capacitively coupled discharges J. Phys. D: Appl. Phys. 41062004

[17] Babaeva N Yu, Lee J K and Kim H C 2004 Non-stationary charging of a dust grain in decaying streamer-channel plasma Plasma Sources Sci. Technol. 13127 